\title{
STUDY OF GRAPHENE-MoS2 BASED SPR BIOSENSOR WITH GRAPHENE BASED SPR BIOSENSOR: COMPARATIVE APPROACH
}

\author{
Md. Mortuza Habib ${ }^{1}$ \\ Ruddro Roy ${ }^{1}$ \\ Md. Mojidul Islam ${ }^{1}$ \\ Mehedi Hassan ${ }^{1}$ \\ Md. Muztahidul Islam ${ }^{1}$ \\ Md. Biplob Hossain ${ }^{2+}$
}

\author{
${ }_{1,2}^{1,2}$ Dept. of Electrical and Electronic Engineering, Bangladesh \\ ${ }^{\prime}$ Bangladesh Army University of Engineering and Technology, Natore, \\ Bangladesh. \\ 'Jashore University of Science and Technology, Jashore, Bangladesh. \\ Email:biplobh.eee10@gmail.com
}

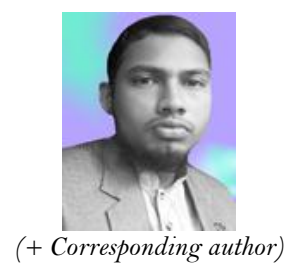

ABSTRACT

\section{Article History}

Received: 24 December 2018 Revised: 21 January 2019 Accepted: 4 March 2019 Published: 29 March 2019

\section{Keywords}

BRE

DNA

SPR

Sensitivity

Graphene

TMDC

MoS2.

\begin{abstract}
In this paper, we compare the sensitivity of graphene-MoS2 based surface plasmon resonance (SPR) biosensor to graphene based SPR biosensor. Here, graphene is used as biomolecular recognition element (BRE) because of its high adsorption ability and optical characteristics which helps to improve sensor sensitivity, on the other hand $\mathrm{MoS} 2$ is used for it has larger band gap, high fluroscence quenching ability, higher optical absorption efficiency which improves further sensor sensitivity. In DNA hybridization event, numerically achieved results show that single layer of graphene$\mathrm{MoS}_{2}$ based SPR biosensor is $175 \%$ more sensitive than single layer of graphene coated SPR biosensor. Surface plasmon resonance angle and spectrum of reflected power are numerically investigated for different concentrated complementary DNA strands. The variations of SPR angle is significantly computable for complementary DNA strands whereas these parameters are varied negligibly for mismatched DNA strands. Thus the proposed sensor effectively differentiates hybridization and single nucleotide polymorphisms (SNP) by examining the level of changes in SPR angle and reflected power spectrum.
\end{abstract}

Contribution/Originality: This study is one of very few studies which have investigated numerically the performance parameters in terms of sensitivity with adding only graphene and other hand adding graphene with MoS2 layers based surface plasmon resonance biosensor.

\section{INTRODUCTION}

Deoxyribonucleic acid or DNA is a long biomolecule composed of matchless genetic instructions for an organism requires building up, being alive, and reproducing. DNA hybridization commonly indicates a biomolecular method that estimates the level of genetic relationship between pools of DNA sequences to obtain the genetic gap between two organisms. This bio technology is being used to catch information of DNA molecular bonding by which more than 400 diseases are directly diagnosable and the number is cumulating day by day [1].

Now a day, Surface Plasmon Resonance (SPR) is broadly being used for detecting biomolecular interactions such as DNA hybridization [2]. Actually, SPR technology is now browbeaten applications in all sides of bioimaging and biosensing. This wide use of SPR technology in current biosensors is because of its good sensing capability, compactness, light weight, ease of multiplexing and reliability not only for DNA hybridization [3-5] but also for other biosensing applications such as enzyme detection [6] protein-protein hybridization [7] proteinDNA hybridization [8, 9] enzyme-substrate or inhibitor hybridization [8, 10] lipid membrane-protein hybridization $[11,12]$ cell or virus-protein hybridization $[13,14]$ and so forth. For the excitation of the surface 
plasmons (SPs) at the metal-dielectric interface, the electrons in the conduction band of SPR active metal must be able to resonate with the incident light of particular wavelength [15]. SPR based prism biosensor has many advantages such as compactness, light weight, high sensitivity, ease of multiplexing and remote sensing and mechanical flexibility So such sensor allows the miniaturization and the chemical or biological sensing [16]; [17].

For SPR biosensor, a thin metallic film ( $\mathrm{Au}$ or $\mathrm{Ag}$ ) is coated around base to separate the sensing medium and the dielectric. Gold film is normally preferred due to its good resistance to oxidation and corrosion in different circumstances. However, for gold based SPR biosensor, biomolecules absorbed poorly on gold, limiting the sensitivity Silver (Ag) coated SPR sensor produces a higher sensitivity than a gold coated SPR sensor but silver is highly susceptible to oxidation [18]. In order to improve the sensitivity of this kind of biosensor, lots of techniques including metal nano-particles and nano-holes [19]; [20] and colloidal gold nano-particles in buffered solution [21] etc., have been proposed.

Graphene is a $2 \mathrm{D}$ nanomaterial which is extensively used due to its superior optical, mechanical and electrical properties, such as high surface to volume ratio, high electron mobility, and stable structure [22, 23]. Grapheneon-Au (111) has been proposed and fabricated very recently, which is shown to detect DNA hybridization in ref [17]. Unlike graphene, other $2 \mathrm{D}$ materials that belongs to the transition-metal dichalcogenide (TMDC) such as molybdenum disulphide (MoS2) which is analogous in structure to graphene, has taken great attentions for its ultra-thin direct thickness-dependent bandgap of $1.3-1.9 \mathrm{eV}$ and semiconductor nature and is therefore promising for designing SPR biosensor of superior sensitivity [24]. This material is already being used in field effect transistor (FET) producing very high charge mobility [25]. The sensitivity of the quantum-confinement-incurred direct band gap in $\mathrm{MoS} 2$ enables the high sensitive detection of bio targets [26]. It also possess high fluorescence quenching ability [27] and different affinity toward ssDNA and double-stranded DNA (dsDNA). Moreover, the surface of $\mathrm{MoS} 2$ is hydrophobic in nature, which is a key property of this material to be used in biological sensing applications, as such surfaces exhibit high affinity of protein-surface adsorption [28]. Chemical vapor deposition (CVD) has made the large area synthesis of MoS2 possible, which has added further in the progress of MoS2-based biosensors [29].

Innumerable numbers of research work have been published by graphene and molybdenum disulphide (MoS2) [28, 29] as (Biomolecular Recognition Element) BRE. Large carrier mobility, large surface area, and large field enhancement at metal-graphene interface have led graphene to be used as substrate in surface plasmon resonance (SPR)-based biosensors, whereas larger band gap, work function, and larger absorption efficiency have given MoS2 an extra edge over graphene. In order to integrate the advantages of both graphene and MoS2, we are motivated to use both of them in a single biosensor which increases the sensitivity.

In this paper, a numerical modeling of highly sensitive Ag-MoS2-Graphene based biosensor is compared to Ag-graphene based SPR biosensor for DNA hybridization detection. A graphene-MoS2 monolayer is sandwiched between silver films and sensing medium as BRE due to graphene and MoS2 have characteristics for enhancing sensor sensitivity. Basically the design process has been carried out by using fourth layer Fresnel's model. The results show that by using single layer of graphene-MoS2 based SPR biosensor enhances 175\% more sensitive than single layer of graphene based SPR biosensor

\section{THEORETICAL DESIGN}

This paper is made known to a numerical configuration shown in Figure 1. SF 11 glass prism with diameter of $\mathrm{D}(50 \mu \mathrm{m})$, sensing region $£(5 \mathrm{~mm})$ are being taken. The base of prism is considered to be coated with a sheet of $\mathrm{Ag}$ film with the thickness of $\mathrm{d}_{2}(40 \mathrm{~nm})$ and then a $\mathrm{MoS}_{2}$-graphene layer is deposited with thickness of $\mathrm{d}_{3}\left(\mathrm{~d}_{3}=\mathrm{M} \times\right.$ $0.65 \mathrm{~nm}$, where $\mathrm{M}$ is the number of $\mathrm{MoS}_{2}$ layer) and thickness of $\mathrm{d}_{4}\left(\mathrm{~d}_{4}=\mathrm{L} \times 0.34 \mathrm{~nm}\right.$, where $\mathrm{L}$ is the number of graphene layers). The reflection $\mathrm{R}$ can be achieved by using the four layer (prism/Ag/Graphen-MoS $2 / \mathrm{sensing}$ medium) model analysis, where first layer is the prism SF 11 glass prism $\left(n_{p}=1.7786\right)$, the second layer is the Ag 
layer which complex dielectric function can be expressed according to the Lorentz-Drude formula in Mak, et al. [24]. The complex refractive index of MoS2 and graphene are $n_{3}=5.9+(0.8) i$ and $n_{4}=3+(1.1487) i$. The final layer is the sensing medium and its refractive index is $\mathrm{n}_{\mathrm{s}}$. The reflected power for p-polarized light is as [17]:

$$
R=\frac{A+\frac{B}{Z_{f}}-z_{i}\left(C+\frac{D}{Z_{f}}\right)}{A+\frac{B}{Z_{f}}+z_{i}\left(C+\frac{D}{Z_{f}}\right)}
$$

\section{Sensing medium}

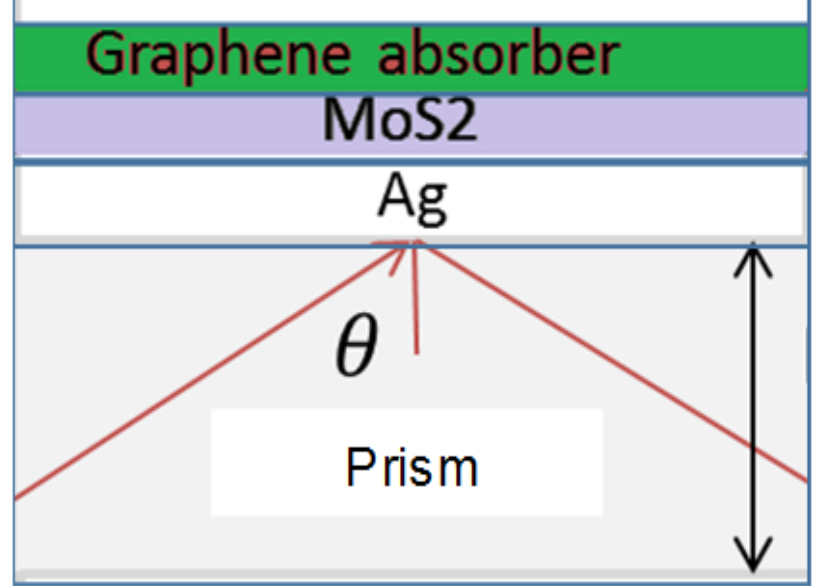

Figure-1. Schematic of the 4-Layered model for SPR biosensor: Prism, Ag (40 nm), MoS2 (.65 $\mathrm{nm})$ and graphene $(0.34 \mathrm{~nm})$ and sensing medium (PBS).

Here, $\mathrm{Zi}$ and $\mathrm{Z}_{\mathrm{f}}$ are initial and final layer wave impedances respectively in the structure of Figure 1 . An individual layer wave impedance can be determined using as Shushama, et al. [17]:

$$
Z_{f}=\frac{K_{s}\left(n_{k} \cos \theta\right)}{\omega \varepsilon_{k}^{2}}
$$

In Equation $2 \mathrm{n}_{\mathrm{k}}$ and $\epsilon_{\mathrm{k}}$ are refractive index (RI) and permittivity of $\mathrm{k}^{\text {th }}$ layer, $\omega=2 \pi / \lambda$ and $\mathrm{k}_{\mathrm{s}}$ is the light wave vector. The variables $\mathrm{A}, \mathrm{B}, \mathrm{C}$ and $\mathrm{D}$ in Equation 1 that can be calculated by solving the following matrix equation as Shushama, et al. [17]:

$$
\left[\begin{array}{ll}
A & B \\
C & D
\end{array}\right]=\left[\begin{array}{cc}
A_{2} & B_{2} \\
C_{2} & D_{2}
\end{array}\right] \times\left[\begin{array}{cc}
A_{3} & B_{3} \\
C_{3} & D_{3}
\end{array}\right] \times \ldots \ldots \times\left[\begin{array}{cc}
A_{N-1} & B_{N-1} \\
C_{N-1} & D_{N-1}
\end{array}\right]
$$

Equation 3 represents generalized interfacial layer system. Here, first layer represents reflectance, therefore they are not present in the matrix equation. In matrix, each element describes the reflection terms of an individual layer. These are the subscripted variables A, B, C and D of each matrix and can be found by the Equation 4 set [17]:

$$
\left.\begin{array}{l}
A_{k}=\cos \left(d_{k} \omega \varepsilon_{k}^{2} \cos \theta_{k}\right) \\
B_{k}=z_{k} \cos \left(d_{k} \omega \varepsilon_{k}^{2} \cos \theta_{k}\right) \\
C_{k}=\frac{\sin \left(d_{k} \omega \varepsilon_{k}^{2} \cos \theta_{k}\right)}{z_{k}} \\
D_{k}=\cos \left(d_{k} \omega \varepsilon_{k}^{2} \cos \theta_{k}\right)
\end{array}\right\}
$$


Here, $d_{k}, \Theta_{k}$ and $Z_{k}$ are the thickness, angle of incidence and wave impedance of $k^{\text {th }}$ layer respectively. The angle of incidence of $\mathrm{k}^{\text {th }}$ layer is unknown that can be found as a function of the refractive index of the initial and $\mathrm{k}^{\text {th }}$ layer as Shushama, et al. [17]:

$$
\theta_{k}=\cos ^{-1}\left(\sqrt{1-\frac{n_{k}}{n_{k+1}} \sin ^{2} \theta}\right)
$$

Light with a wavelength of $633 \mathrm{~nm}$ is emitted from a monochromatic source, and the corresponding data are collected with a spectrometer and computer. At this time, incident wave propagates continuously along the horizontal direction as shown in Figure 1, with wave vector of following equation [1]; [8]:

$$
k_{\text {light }}=2 \pi / \lambda\left(n_{p} \sin \theta\right)
$$

Equation 6 represents the ingredient of light propagating parallel to the silver surface. The direct incidence of TM polarized light cannot excite SPW at the metal dielectric interface because of its relatively small wave vector. Here, $\theta$ is the angle of incidence of the light with the metal surface and $n_{p}$ is the refractive index (RI) of the prism. In the dielectric sensing layer, a variation of molecules concentration will produce small change in the refractive index near the metal interface. This variation of refractive index will eventually change the wave vector of the surface plasmon waves $\left(\mathrm{k}_{\mathrm{SPW}}\right)$, which can be measured by attenuated total reflection (ATR) method. The surface plasmon wave (SPW) wave vector, $k_{s p w}$ is described in Equation 7 , where $n_{t g}$ is the RI of the gold and $n_{g}$ is the RI of the graphene [1]; [9]:

$$
k_{S P W}=\frac{2 \pi}{\lambda} \sqrt{\frac{n_{A g}^{2} n_{g}^{2}}{n_{A g}^{2}+n_{g}^{2}}}
$$

The majority of SPR applications connect with the real RI changes due to chemical or biochemical action [1] and therefore, Equation 8 is formed by considering real quantities only. A resonant excitation of photon-electron coupling takes place when the wave vector of the incident light matches that of the SPW (Surface plasmon wave), these two are equal $\left(k_{\text {light }}=k_{\mathrm{SPR}}\right.$ ), which produces shift of incident angle. The total reflectance vs angle of incidence or reflectance vs wavelength characteristics curve is known as the SPR curve. The incident angle at plasmon resonance is called SPR angle that can be represented in ref [11] as:

$$
\theta_{S P R}=a \sin \sqrt{\frac{n_{A g}^{2} n_{s}^{2}}{n_{p}^{2}\left(n_{A g}^{2}+n_{s}^{2}\right)}}
$$

The reflectance spectra show a dip at the resonance condition when the wave vector of the incident light matches with the SPW.

\section{RESULTS AND DISCUSSIONS}

The refractive index $(\mathrm{RI})$ of the sensing medium changes due to submersing probe DNA (p-DNA) in the sensing medium. The changed refractive index $\left(n_{s}^{2}\right.$ in Equation 9$)$ of sensing medium after adsorption of DNA molecules is mathematically expressed as Hossain and Rana [3]:

$$
n_{s}^{2}=n_{s}^{1}+c_{a} \frac{\partial n_{s}}{\partial c_{a}}
$$

Where, $c_{\mathrm{a}}$ is the concentration of adsorbed molecules, $n^{i}$ is the RI of the sensing medium before adsorption of

DNA molecules and $\frac{d n}{d c}$ is the increment in refractive index due to the adsorbate. The refractive index increment 
parameter is $\frac{d n}{d c}=0.182 \mathrm{~cm}^{3} / \mathrm{gm}$ in the case of using water. DNA molecules shift the SPR angle rightwards due to the addition of probe which changes the refractive index of the sensing dielectric.

By introduction of DNAs as electron-rich molecules, the number of carriers should change in the graphene or $\mathrm{MoS}_{2}$-graphene layer concentration which has led in altering the propagation constant. Thus the proposed SPR sensor with high sensitivity is applied to detect the DNA hybridization based on the SPR angle variations. Sensing probe DNA hybridization by the proposed SPR biosensors are shown in Figure 2. The results show that SPR angle is 74.4 degree after adding probe DNA in graphene coated SPR biosensor and SPR angle is 76.40 degree after adding probe DNA in $\mathrm{MoS}_{2}$-graphene hybrid based SPR sensor. It can be seen in Figure 2 that SPR curve shows ambipolar behavior since the SPR angle can be controlled by the refractive index.

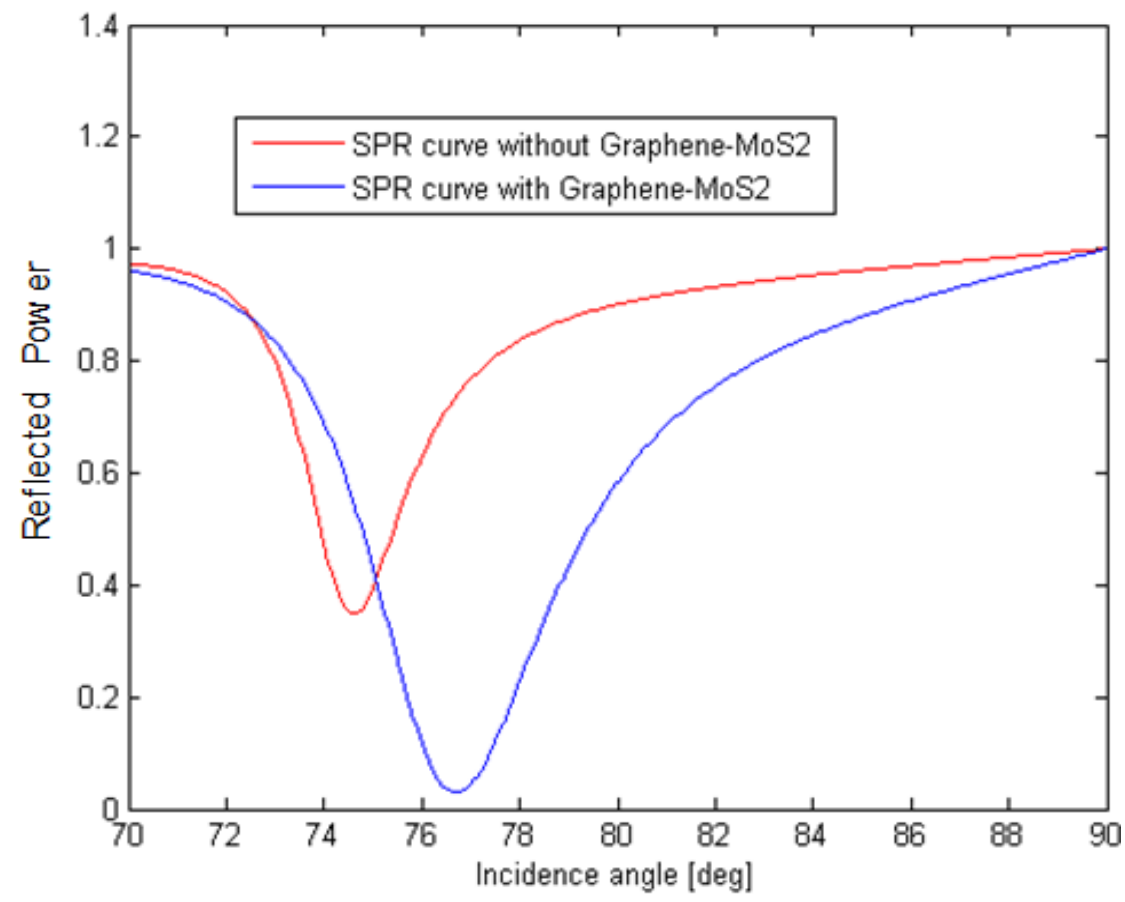

Figure-2. SPR curve for with graphene-MoS2 and without graphene graphene-MoS2 layer.

Complementary hybridization event is defined when two single-stranded complementary ssDNA, one is probe and the rest is target, is attached together and form double-stranded (dsDNA) helix structure. The proposed model explains the analytical behavior of the sensor to detect the hybridization of target DNAs to the probe DNAs that are pre-immobilized on $\mathrm{MoS}_{2}$-graphene surface.

Figure $3(\mathrm{a})$ and Figure $3(\mathrm{~b})$ describe the $R-\theta$ characteristic of the proposed model for different concentrations of complementary DNA, where each diagram depicts specific concentration of the DNA molecules. In Figure 3(a) and Figure 3(b) a four layer prism-Ag-Graphene-Sensing layer and prism-Ag-MoS ${ }_{2}$-GrapheneSensing layer composed of complementary DNA, probe DNA and sensing medium.

The corresponding SPR curves after adding only $1000 \mathrm{nM}$ probe DNA, after adding $1000 \mathrm{nM}, 1001 \mathrm{nM}, 1010$ $\mathrm{nM}$, and $1100 \mathrm{nM}$ of complementary DNA are shown in Figure 3. According to these analytical data, one important factor, detection parameter which is the SPR angle, plays crucial role in detecting the DNA hybridization events. Table 1 and Table 2 gives us information about how $\theta_{\text {SPR }}$ and $R_{\min }$ parameters change for Ag-Graphene hybrid based and $\mathrm{Ag}-\mathrm{MoS}_{2}-$ Graphene hybrid based SPR sensor respectively with different concentrations of complementary DNA molecules which reveals the sensitivity, $\theta_{\mathrm{SPR}}$ towards the hybridization of the target DNAs. 

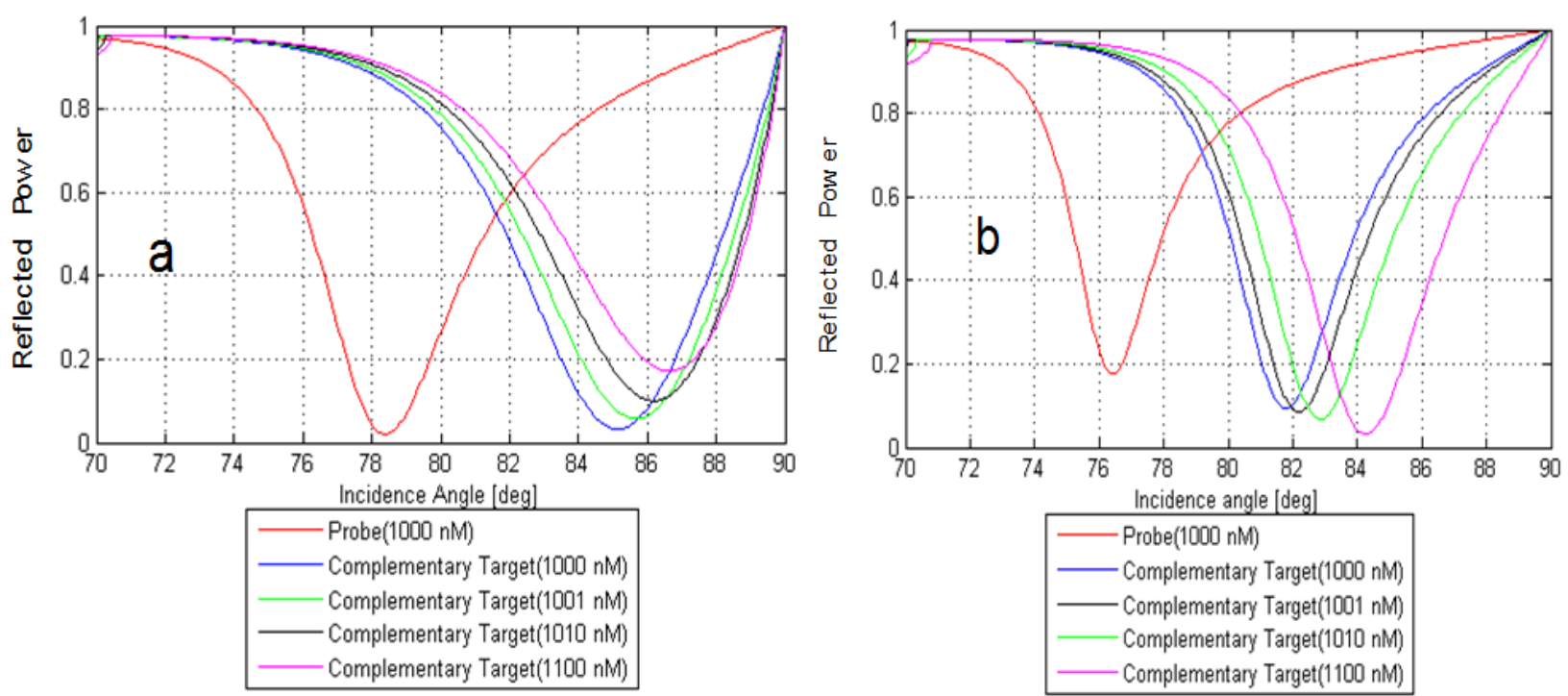

Figure -3. Sensing probe DNA and complementary DNA hybridization by the proposed (a) Ag-Graphene hybrid based and (b) Ag-MoS ${ }_{2}-$ Graphene hybrid based SPR curves of DNA molecules after adding different complementary DNA

It is apparently seen that the considerable increase of SPR angle is a sign of probe-target matching combination in DNA hybridization. The analytical data indicates the strong dependency of the SPR angle on the concentration increment which can have a predictable influence on the reflectance-incident angle characteristics of SPR device.

Table-1. Rmin and $\theta_{\mathrm{SPR}}$ for Different Concentration of DNA Molecules for Ag-Graphene hybrid based SPR sensor

\begin{tabular}{l|c|c}
\hline Concentration $\left(\mathbf{c}_{\mathbf{a}}\right)[\mathbf{n M}]$ & $\mathbf{R}_{\mathrm{MIN}}$ & $\boldsymbol{\theta}_{\text {SPR }}[\mathbf{d e g}]$ \\
\hline 1000 (Probe) & 0.1777 & 76.40 \\
\hline 1000 (Complementry Target) & 0.0943 & 81.80 \\
\hline 1001 (Complementry Target) & 0.0849 & 82.20 \\
\hline 1010 (Complementry Target) & 0.0682 & 82.85 \\
\hline 1100 (Complementry Target) & 0.0321 & 84.25 \\
\hline
\end{tabular}

Table-2. Rmin and $\theta_{\mathrm{SPR}}$ for Different Concentration of DNA Molecules for Ag-MoS2-Graphene hybrid based SPR sensor.

\begin{tabular}{l|c|c}
\hline Concentration $\left(\mathbf{c}_{\mathbf{a}}\right)[\mathbf{n M}]$ & $\mathbf{R}_{\mathrm{MIN}}$ & $\boldsymbol{\theta}_{\text {SPR }}[\mathrm{deg}]$ \\
\hline $1000(\mathrm{P}$ obe) & 0.0204 & 78.40 \\
\hline 1000 (Complementry Target) & 0.0324 & 85.15 \\
\hline 1001 (Complementry Target) & 0.0573 & 85.65 \\
\hline 1010 (Complementry Target) & 0.1001 & 86.20 \\
\hline 1100 (Complementry Target) & 0.1718 & 86.65 \\
\hline
\end{tabular}

The main performance parameters of the SPR sensor which are characterized on the basis of its sensitivity should as high as possible for a good sensor [28]. The sensitivity $(\mathrm{S})$ is defined as the ratio of shift in the SPR angle of incidence $\left(\Delta \theta_{S P R}^{T}\right)$ to the change in measure and, e.g. moles of biomolecules $\left(\Delta \mathrm{c}_{\mathrm{a}}\right)$ as following [3] in Equation 10:

$$
s=\frac{\Delta \theta_{S P R}}{\Delta c_{a}}
$$

Table-3. $\mathrm{R}_{\min }, \theta_{\mathrm{SPR}}$ and $\Delta \theta_{\mathrm{SPR}}$, for different layers

\begin{tabular}{l|c|c|c}
\hline Graphene (L)/MoS2(N) layer & $\mathbf{R}_{\min }$ & $\boldsymbol{\theta}_{\text {SPR }}[\mathbf{d e g}]$ & $\Delta \theta_{\text {SPR }}[\mathbf{d e g}]$ \\
\hline $\mathrm{L}=0 \& \mathrm{M}=0$ (Conventional) & 0.3484 & 74.60 & 0.00 \\
\hline $\mathrm{L}=1 \& \mathrm{M}=0$ (Graphene Layer) & 0.1883 & 74.95 & 0.35 \\
\hline $\mathrm{L}=1 \& \mathrm{M}=1($ MoS2-Graphene coated) & 0.0293 & 76.70 & 2.10 \\
\hline
\end{tabular}


The change of SPR angle $\left(\Delta \theta_{\mathrm{SPR}}\right)$ and minimum reflected power $\left(\mathrm{R}_{\min }\right)$ due to the DNA hybridization, has been compared among conventional $(\mathrm{L}=0$ \& $\mathrm{M}=0)$, Graphene Layer $(\mathrm{L}=1 \& \mathrm{M}=0)$ and $\mathrm{MoS} 2-\mathrm{Graphene}$ coated $(\mathrm{L}=1 \&$ $\mathrm{M}=1$ ) respectively. These values are tabulated in Table 3.

Sensitivity analysis for graphene based SPR sensor is given in Equation 11. Here, $S^{0}$ is the sensitivity without graphene, $S^{L}$ is the sensitivity with graphene. Similar way sensitivity for graphene-MoS2 hybrid based SPR sensor is calculated by incorporating Equation 12 . Here, $S^{L, M}$ is the sensitivity after adding graphene-MoS2. Result from Table 3 and Equation 11 and 12 shows that by using single graphene layer which provides 35\% more sensitivity compared to conventional sensor. Whereas by using single MoS2-graphene layer which provides $210 \%$ more sensitivity compared to conventional sensor and $175 \%$ more sensitive than graphene coated SPR sensor.

$$
\begin{aligned}
& S^{L}=\frac{\Delta \theta^{T} S P R}{\Delta c_{a}}=\frac{\Delta \theta^{0} S P R+\Delta \theta^{L} S P R}{\Delta c_{a}} \\
&= \frac{\Delta \theta^{0} S P R+.35 \Delta \theta^{0} S P R}{\Delta c_{a}} \\
&= \frac{(1+0.35 L) \Delta \theta^{0} S P R}{\Delta c_{a}} \\
&=(1+0.35 L) s^{O} \\
& S^{L-M}=\frac{\Delta \theta^{T} S P R}{\Delta c_{a}}=\frac{\Delta \theta^{0} S P R+\Delta \theta^{L-M} S P R}{\Delta c_{a}} \\
&=\frac{\Delta \theta^{0} S P R+2.10 \Delta \theta^{0} S P R}{\Delta c_{a}} \\
&=\frac{[1+2.10(M \times L)] \Delta \theta^{0} S P R}{\Delta c_{a}} \\
&=[1+2.10(M \times L)] S^{O}
\end{aligned}
$$

\section{CONCLUSIONS}

In this work the performance parameters in terms of sensitivity with adding only graphene and other hand sandwiching graphene-MoS2 layers have been numerically described. Then a comparison study has been made between them. It has been seen that single layer graphene is added then sensitivity is increased by $35 \%$. But when graphen-MoS2 hybrid is added then sensitivity is increased by $210 \%$ which is more $175 \%$ sensitive than graphene coated fiber optic SPR biosensor.

Funding: This work was supported by the Dept. of Electrical and Electronic Engineering both at Rajshahi University of Engineering and Technology and Jashore University of Science and Technology, Bangladesh. Competing Interests: The authors declare that they have no competing interests.

Contributors/Acknowledgement: All authors contributed equally to the conception and design of the study.

\section{REFERENCES}

[1] K. Tamersit and F. Djeffal, "Double-gate graphene nanoribbon field-effect transistor for DNA and gas sensing applications: Simulation study and sensitivity analysis," IEEE Sensors Journal, vol. 16, pp. 4180-4191, 2016. Available at: https://doi.org/10.1 109/jsen.2016.2550492.

[2] M. Hossain and M. Rana, "Graphene coated high sensitive surface plasmon resonance biosensor for sensing DNA hybridization," Sensor Letters, vol. 14, pp. 145-152, 2016. Available at: https://doi.org/10.1166/sl.2016.3596.

[3] M. B. Hossain and M. M. Rana, "DNA hybridization detection based on resonance frequencyreadout in graphene on Au SPR biosensor," Journal of Sensors, vol. 2016, pp. 1-7, 2016. 
A. I. K. Lao, X. Su, and K. M. M. Aung, "SPR study of DNA hybridization with DNA and PNA probes under stringent conditions," Biosensors and Bioelectronics, vol. 24, pp. 1717-1722, 2009. Available at: https://doi.org/10.1016/j.bios.2008.08.054.

H. Fu, S. Zhang, H. Chen, and J. Weng, "Graphene enhances the sensitivity of fiber-optic surface plasmon resonance biosensor," IEEE Sensors Journal, vol. 15, pp. 5478-5482, 2015. Available at: https://doi.org/10.1109/jsen.2015.2442276.

[6] M. Pumera, "Graphene in biosensing," Materials Today, vol. 14, pp. 308-315, 2011.

[7] A. Madeira, E. Vikeved, A. Nilsson, B. Sjögren, P. E. Andrén, and P. Svenningsson, "Identification of protein-protein interactions by surface plasmon resonance followed by mass spectrometry," Current Protocols in Protein Science, vol. 65, pp. 19.21. 1-19.21. 9, 2011 . Available at: https://doi.org/10.1002/0471140864.ps1921s65.

J. Majka and C. Speck, "Analysis of protein-DNA interactionsusing surface plasmon resonance," Advances in Biochemical Engineering/Biotechnology, vol. 104, pp. 13-36, 2007. Available at: https://doi.org/10.1007/10_026.

H. F. Teh, W. Y. Peh, X. Su, and J. S. Thomsen, "Characterization of protein- DNA interactions using surface plasmon resonance spectroscopy with various assay schemes," Biochemistry, vol. 46, pp. 2127-2135, 2007. Available at: https://doi.org/10.1021/bio61903t.

[10] C.-C. Fong, W.-P. Lai, Y.-C. Leung, S. C.-L. Lo, M.-S. Wong, and M. Yang, "Study of substrate-enzyme interaction between immobilized pyridoxamine and recombinant porcine pyridoxal kinase using surface plasmon resonance biosensor," Biochimica et Biophysica Acta, vol. 1596, pp. 95-107, 2002. Available at: https://doi.org/10.1016/s01674838(02)00208-x.

[11] O. L. B. Acevedo and D. Pauron, "Protein-lipid interaction analysis by surface plasmon resonance (SPR)," Bio-Protocol, vol. 4, pp. 1-8, 2014. Available at: https://doi.org/10.21769/bioprotoc.1237.

[12] E. M. Erb, X. Chen, and S. Allen, "Characterization of thesurfaces generated by liposome binding to the modiied dextran matrix of a surface plasmon resonance sensor chip," Analytical Biochemistry, vol. 280, pp. 29-35, 2000. Available at: https://doi.org/10.1006/abio.1999.4469.

[13] M. Beseničar, P. Maček, J. H. Lakey, and G. Anderluh, "Surface plasmon resonance in protein-membrane interactions," Chemistry and Physics of Lipids, vol. 141, pp. 169-178, 2006. Available at: https://doi.org/10.1016/j.chemphyslip.2006.02.010.

[14] H. Miyoshi, N. Suehiro, K. Tomoo, S. Muto, T. Takahashi, T. Tsukamoto, T. Ohmori, and T. Natsuaki, "Binding analyses for the interaction between plant virus genome-linked protein (VPg) and plant translational initiation factors," Biochimie, vol. 88, pp. 329-340, 2006. Available at: https://doi.org/10.1016/j.biochi.2005.09.002.

[15] J. Homola, "Present and future of surface plasmon resonance biosensors," Analytical and Bioanalytical Chemistry, vol. 377, pp. 528-539, 2003. Available at: https://doi.org/10.1007/s002 16-003-2 101-0.

[16] J. L. E. Ronald, "Multiwavelength surface plasmon resonance sensor designs for chemical and biochemical detection," P.H.D. Thesis, Dept. of Chemistry, Virginia Polytechnic Institute and State University, Ontario, Canada, 2013.

[17] K. N. Shushama, M. M. Rana, R. Inum, and M. B. Hossain, "Graphene coated fiber optic surface plasmon resonance biosensor for the DNA hybridization detection: Simulation analysis," Optics Communications, vol. 383, pp. 186-190, 2017. Available at: https://doi.org/10.1016/j.optcom.2016.09.015.

[18] B. H. Ong, X. Yuan, S. C. Tjin, J. Zhang, and H. M. Ng, "Optimised film thickness for maximum evanescent field enhancement of a bimetallic film surface plasmon resonance biosensor," Sensors and Actuators B: Chemical, vol. 114, pp. 1028-1034, 2006. Available at: https://doi.org/10.1016/j.snb.2005.07.064.

[19] L. J. Sherry, R. Jin, C. A. Mirkin, G. C. Schatz, and R. P. Van Duyne, "Localized surface plasmon resonance spectroscopy of single silver triangular nanoprisms," Nano Letters, vol. 6, pp. 2060-2065, 2006. Available at: https://doi.org/10.1021/nlo61286u.

[20] M. E. Stewart, C. R. Anderton, L. B. Thompson, J. Maria, S. K. Gray, J. A. Rogers, and R. G. Nuzzo, "Nanostructured plasmonic sensors," Chemical Reviews, vol. 108, pp. 494-52 1, 2008. Available at: https://doi.org/10.1021/cr068126n. 
[21] P. Englebienne, A. V. Hoonacker, and M. Verhas, "Surface plasmon resonance: Principles, methods and applications in biomedical sciences," Journal of Spectroscopy, vol. 17, pp. 255-273, 2003. Available at: https://doi.org/10.1155/2003/372913.

[22] J. Kim, H. Son, D. J. Cho, B. Geng, and W. Regan, "Electrical control of optical plasmon resonance with graphene," Nano Letters, vol. 12, p. 5598-5602, 2012. Available at: https://doi.org/10.1021/nl302656d.

[23] M. Hossain, S. Muktadhir, and M. M. Rana, "Multi-structural optical devices modeling using graphene tri-layer sheets," Optik, vol. 127, pp. 5841-5851, 2016. Available at: https://doi.org/10.1016/j.ijleo.2016.03.075.

[24] K. F. Mak, C. Lee, J. Hone, J. Shan, and T. F. Heinz, "Atomically thin MoS2: A new direct-gap semiconductor," Physical Review Letters, vol. 105, p. 136805, 2010. Available at: https://doi.org/10.1 103/physrevlett.105.136805.

[25] O. Lopez-Sanchez, D. Lembke, M. Kayci, A. Radenovic, and A. Kis, "Ultrasensitive photodetectors based on monolayer MoS2," Nature Nanotechnology, vol. 8, pp. 497-501, 2013. Available at: https://doi.org/10.1038/nnano.2013.100.

[26] W. Bao, X. Cai, D. Kim, K. Sridhara, and M. S. Fuhrer, "High mobility ambipolar MoS2 field-effect transistors: Substrate and dielectric effects," Applied Physics Letters, vol. 102, pp. 042104-4 2012, 2013. Available at: https://doi.org/10.1063/1.4789365.

[27] K. Rurack, "Flipping the light switch 'ON'-the design of sensor molecules that show cation-induced fluorescence enhancement with heavy and transition metal ions," Spectrochimica Acta Part A: Molecular and Biomolecular Spectroscopy, vol. 57, pp. 2161-2 195, 2001. Available at: https://doi.org/10.1016/s1386-1425(01)00492-9.

[28] A. B. Farimani, K. Min, and N. R. Aluru, "DNA base detection using a single-layer MoS2," ACS Nano, vol. 8, pp. 79147922, 2014. Available at: https://doi.org/10.1021/nn5029295.

[29] A. Castellanos-Gomez, N. Agraït, and G. Rubio-Bollinger, "Optical identification of atomically thin dichalcogenide crystals," Applied Physics Letters, vol. 96, p. 213116,2010 . Available at: https://doi.org/10.1063/1.3442495.

Views and opinions expressed in this article are the views and opinions of the author(s), International Journal of Natural Sciences Research shall not be responsible or answerable for any loss, damage or liability etc. caused in relation to/arising out of the use of the content. 